\title{
Imaging Findings of Multiphase CT Angiography of Acute Internal Carotid Artery Occlusion Within 6-Hour Time-Window After Thrombectomy and Its Clinical Implication
}

\author{
Chun-Chao Huang ${ }^{1}$, Chao-Liang Chou ${ }^{2}$, Wei-Ming Huang ${ }^{1}$, Zong-Yi Jhou ${ }^{1}$, Yung-Pin Hwang ${ }^{2}$, Hsin-Yao \\ Lin $^{3}$, Yuan-Hsiung Tsai ${ }^{4}$ and Chun-Hsien Lin $^{5,}{ }^{*}$ \\ ${ }^{1}$ Department of Radiology, MacKay Memorial Hospital, Taipei, Taiwan \\ ${ }^{2}$ Department of Neurology, Mackay Memorial Hospital, Taipei, Taiwan \\ ${ }^{3}$ Division of Neurosurgery, Department of Surgery, MacKay Memorial Hospital, Taipei, Taiwan \\ ${ }^{4}$ Department of Diagnostic Radiology, Chang Gung Memorial Hospital, Chiayi, Taiwan \\ ${ }^{5}$ Department of Neurology, Chang Gung Memorial Hospital, Chiayi, Taiwan \\ "Corresponding author: Department of Neurology, Chang Gung Memorial Hospital, Pu Road, Chiayi, Taiwan. Tel: +886-53621000; 2759, Fax: +886-53623002, Email: \\ dopamine32@gmail.com
}

Received 2020 March 25; Revised 2020 July 22; Accepted 2020 July 26.

\begin{abstract}
Background: Intra-arterial thrombectomy (IAT) is one of the mainstream treatments for acute ischemic stroke. As relatively little evidence on extracranial internal carotid artery (ICA) occlusions exists in the literature, we share our experiences after using IAT for intracranial and extracranial ICA occlusions. We further clarify the imaging characteristics of multiphase computed tomography angiography (CTA) and share the experience of balloon angioplasty in acute ICA occlusion.

Objectives: To investigate the imaging findings of multiphase CTA and the clinical conditions and outcomes of acute ICA occlusions at different segments after IAT.

Patients and Methods: All participants receiving IAT for acute stroke with isolated ICA occlusions were included, obtained from three hospitals between June 2016 and November 2018. An imaging review for non-enhanced computed tomography (CT), multiphase CTA, and angiography was conducted. Epidemiological and clinical data were reviewed. Further comparisons were evaluated between the occlusion side from the $\mathrm{C} 6$ to $\mathrm{C} 7$ segments and $\mathrm{C} 1$ to $\mathrm{C} 5$ segments of the ICA.

Results: The average age of the patients was 73.0 years, and the initial National Institutes of Health Stroke scale (NIHSS) score was 18.6. Nineteen percent of cases had good outcomes. Good reperfusion results were achieved in $50 \%$ of cases. Compared to the group with occlusion from C6-C7, the group with occlusion from C1-C5 had a relatively good Alberta Stroke Program Early CT score (ASPECTS) in the A1 phase of the multiphase CTA, and more patients had a good collateral. Three cases received a balloon angioplasty for a concurrent proximal cervical ICA tight stenosis, and all cases had good reperfusion results.

Conclusion: Distal occlusion of the ICA demonstrates lower ASPECTS and a worse collateral on multiphase CTA. A multiphase CTA with an extended scan range in the $\mathrm{A} 2$ and $\mathrm{A} 3$ phases is able to exclude pseudo-occlusion of the ICA. Balloon angioplasty is helpful for concurrent proximal cervical ICA tight stenosis.
\end{abstract}

Keywords: Intra-Arterial Thrombectomy, Internal Carotid Artery Occlusion, Multiphase CTA, Pseudo-Occlusion, Balloon Angioplasty

\section{Background}

Intra-arterial thrombectomy(IAT) has been considered as a standard treatment for acute strokes with large vessel occlusions that occur within reasonable time windows. IAT became the standard after publication of the five large randomized trials in 2015 (1-5) and was included in the American Heart Association and American Stroke Association treatment guidelines for acute stroke in 2018 (6). A metaanalysis reported that IAT improved outcomes, defined as a modified Rankin scale (mRS) score from 0 to 2 within 3 months after an acute stroke with large vessel occlusion. This was seen in $46.0 \%$ of patients, compared to the control group, in which only $26.5 \%$ had good outcomes. Besides that, there were no significant differences in intracranial hemorrhage or mortality between the IAT and control groups (7). In the interventional group, most cases had an occlusion location in the middle cerebral artery (77\%), mainly at the M1 segment (69\%), with the internal carotid 
artery (ICA) location occupying $21 \%$ of all cases, mainly the distal ICA location. For these five large randomized trials in 2015, the imaging inclusion criteria relied on nonenhanced computed tomography (CT), single-phase CT angiography (CTA), magnetic resonance imaging (MRI), or angiographic results (1-5). Meanwhile, multiphase CTA was invented as a new imaging tool for acute stroke evaluation, which has as much power as CT perfusion but also a lower radiation dose and no need for additional contrast material injection or further post-processing at an independent workstation (8). In this study, we report our experience with the different imaging results, including multiphase CTA, and their clinical implications for acute stroke patients with ICA occlusions in the intracranial and extracranial segments that are receiving IAT. Further discussion was focused on the imaging characteristics of multiphase CTA in acute ICA occlusion and the role of balloon angioplasty for concurrent proximal ICA tight stenosis in IAT. Moreover, the clinical and imaging outcomes were compared between the distal and proximal ICA occlusion groups.

\section{Objectives}

To investigate the imaging findings of multiphase CTA and the clinical conditions and outcomes of acute ICA occlusions at different segments after IAT.

\section{Patients and Methods}

\subsection{Patients}

All participants who received IAT for acute stroke in three hospitals between June 2016 and November 2018, whose occlusion arteries were angiographically proven to be ICA were included in this study. An imaging review was conducted, and patients with the presence of tandem lesions of additional intracranial occlusions were excluded. The epidemiological and clinical data were reviewed based on the medical records, registration system, and imaging system. Patients without available non-enhanced CT or multiphase CTA were excluded from this study. The epidemiological data included age, sex, risk factors for stroke, and antithrombotic agent usage.

\subsection{Clinical Data}

The included parameters during the acute stroke management were initial National Institutes of Health Stroke scale (NIHSS) scores, tissue plasminogen activator infusion, and mRS scores within 3 months. The NIHSS is a 15item neurologic examination scale for acute cerebral infarction. The overall score ranged from 0 , which was normal, to 42, which indicated the highest impairment score.
The score was evaluated by certified neurologists. The mRS scores were used to evaluate the degree of disability of patients after stroke. The higher the score, the more severely disabled was the patient. The scale ranged from 0 , which indicated that they had no symptoms, to 6 , which indicated that they were dead. Usually, a better outcome was defined as an mRS score from 0 to 2 , which indicated slight disability.

\subsection{Radiographic Results}

The imaging results of all patients, including nonenhanced CT, multiphase CTA, and angiography, were retrospectively evaluated by a single neuroradiologist with 7 years of experience, in order to diminish the inter-observer bias.

The Alberta Stroke Program Early CT scores (ASPECTS) were measured using non-enhanced CT. The middle cerebral artery territory of the affected cerebral hemisphere was divided into 10 regions, and the normal, full score was a score of 10 points. If an acute ischemic change was suspected for any of them, their score would be subtracted by one point; the minimum score was zero, which indicated that all the middle cerebral artery territories were affected. Generally, ASPECTS with at least 6 points was considered to be related to a better outcome. There were four series of images in a multiphase CTA, including pre-contrast images, first arterial phase (A1) images, second arterial phase (A2) images, and third arterial phase (A3) images. ASPECTS scores were also measured in pre-contrast and A1 images of multiphase CTAs. The occlusion side of ICA was recorded based on the multiphase CTAs. ICA was divided into seven segments, including C1 (cervical segment), C2 (petrous segment), C3 (lacerum segment), C4 (cavernous segment), C5 (clinoid segment), C6 (ophthalmic segment), and C7 (communicating segment). The predicted occlusion segment of ICA was defined as the most distal enhanced segment or the most proximal non-enhanced segment if there was no contrast enhancement of ICA in the first image. The predicted occlusion segment was evaluated in A1 and A3 images. Whether there was contrast migration in multiphase CTA was based on the difference of the predicted occlusion segments between A1 and A3 images. The collateral grading of multiphase CTA was measured according to the original article of the development of multiphase CTA (8). The collateral grading system included 0 to 5 collateral grades, where a grade of 4 and 5 represented an intermediate and good collateral, respectively. A grade between 0 and 3 represented a poor collateral.

For IAT angiography, we collected data on the relative use of general anesthesia, final occlusion segment of ICA, usage of balloon angioplasty for concurrent proximal cervical ICA stenosis, conduction times of suction and 
stent-retrieving, onset-to-reperfusion time, and modified thrombolysis in cerebral infarction (mTICI) grade. The mTICI grade was used to evaluate reperfusion outcome after IAT, ranging from grade 0 , meaning no reperfusion, to grade 3, meaning complete reperfusion. Grade 1 was minimal reperfusion. Grade 2 was obvious reperfusion, but there was still a presence of occluded branches, which was divided into grade $2 \mathrm{a}$ and $2 \mathrm{~b}$. Grade $2 \mathrm{a}$ was reperfusion of less than half of the affected territory, and grade $2 b$ was reperfusion of more than half of the affected territory. The mTICI grade $2 \mathrm{~b}$ or 3 was considered as a good reperfusion result.

\subsection{Statistical Analysis}

The statistical analysis was performed using SPSS software, version 24.0 (IBM, Armonk, NY). A descriptive analysis for all parameters was conducted to display numbers and percentages. The final occlusion site of the affected ICA was divided into two groups. The first group had an ICA occlusion at the $\mathrm{C} 6$ or $\mathrm{C} 7$ segment. The other group had an ICA occlusion between $\mathrm{C} 1$ and $\mathrm{C} 5$ segments. A t-test was used to compare NIHSS and ASPECTS in non-enhanced CT, precontrast, and A1 phases of multiphase CTA, as well as collateral grading between these two groups. A non-parametric statistical test was used to evaluate the differences between the percentage of patients with a good collateral grade and good mTICI score, and the percentage of those with good 3month mRS between these two groups. A p-value less than 0.05 was considered statistically significant.

\section{Results}

There were 32 cases included in this study. The average age was $73.0 \pm 2.3$ years, ranging from 34 to 95 , and there were 17 women and 15 men. Among all, there were 23 cases with hypertension, 11 cases with diabetes, 20 cases with hyperlipidemia, eight cases with a history of smoking, 21 cases with atrial fibrillation, and 17 cases being treated with antithrombotic agents. The average of the initial NIHSS scores was $18.6 \pm 0.9$, ranging from 8 to 30 . Sixteen cases had received tissue plasminogen activator treatment. According to the follow-up record for the 3-month mRS score, there were six cases with scores of 0 to 2 . The detailed information can be found in Table 1 .

There were 14 cases with a right ICA occlusion and 18 cases with a left ICA occlusion. The average of the ASPECTS scores in the non-enhanced brain CTs was $8.6 \pm 0.3$, ranging from 4 to 10 . The averages for the pre-contrast and A1 images of the multiphase CTAs were $7.1 \pm 0.5$ and $5.0 \pm 0.6$, respectively. The details of the predicted occlusion ICA segments in A1 and A3 images of the multiphase CTA are listed

\begin{tabular}{ll}
\hline Table 1. Patient Demographics & \\
\hline Variable & \\
\hline Age, $\mathbf{y}$ & $73.0 \pm 2.3(34-95)$ \\
Female & $17(53.1)$ \\
\hline Risk factors & \\
\hline \multicolumn{1}{c}{ Hypertension } & $23(71.9)$ \\
\hline \multicolumn{1}{c}{ Diabetes } & $11(34.4)$ \\
\hline \multicolumn{1}{c}{ Hyperlipidemia } & $20(62.5)$ \\
\hline \multicolumn{1}{c}{ Smoking } & $8(25.0)$ \\
\hline \multicolumn{1}{c}{ Atrial fibrillation } & $21(65.6)$ \\
\hline Antithrombotic agent usage & $17(53.1)$ \\
\hline NIHSS & $18.6 \pm 0.9(8-30)$ \\
\hline tPA treatment & $16(50.0)$ \\
\hline 3-month-mRS & $0: 1,1: 3,2: 2,3: 5,4: 9,5: 2,6: 10(0-2: 19 \%)$ \\
\hline
\end{tabular}

Abbreviations: mRS, modified Rankin scale; NIHSS, National Institutes of Health Stroke Scale; SD, standard deviation; tPA, tissue plasminogen activator. ${ }^{\mathrm{a}}$ Values are expressed as mean $\pm \mathrm{SD}$ (range) or No. (\%).

in Table 2. There were 12 cases with contrast migration in at least one segment, based on the A1 to A3 images. For the other 20 cases without contrast migration, there were 18 cases with stable contrast stasis in their $\mathrm{C} 1$ segments. According to the collateral grading method, there were six cases with intermediate collateral and six with good collateral. For the angiographic results, 17 cases received IAT under general anesthesia. The occlusion segments seen on the angiography images are detailed in Table 2. Only three cases required a balloon angioplasty for a concurrent tight stenosis of the proximal cervical ICA, and these three cases also had contrast stasis at the $\mathrm{C} 1$ segment on their multiphase CTA. Each procedure included suction an average of 2.3 times and stent-retrieving 0.8 times. The average onset-to-reperfusion time was $338.5 \pm 15.7$ minutes, ranging from 215 to 594 minutes. The final IAT results showed mTICI scores of $2 b$ to 3 in 16 cases. All the detailed information related to the non-enhanced CTs, multiphase CTAs, and angiographies are listed in Table 2.

The patients with an angiographic occlusion segment at $\mathrm{C} 6$ and $\mathrm{C} 7$ were compared to the rest of the patients, and the results are listed in Table 3. The significant differences between these two groups included lower ASPECTS in the A1 images in the C6-7 occlusion group, and less patients with good collateral in the C6-7 occlusion group.

In addition, the results of patients with contrast migration on multiphase CTA were compared to those without contrast migration. There were two significant differences. First, the contrast migration group had a lower ASPECTS on the A1 images ( $3.3 \pm 2.9$, range: $0-8)$, compared with the 


\begin{tabular}{|c|c|}
\hline Variable & Values $(n=32)$ \\
\hline ASPECTS & $8.6 \pm 0.3(4-10)(\geq 6: 91 \%)$ \\
\hline Right side occlusion & $14(43.8)$ \\
\hline mCTA-Pre-ASPECTS & $7.1 \pm 0.5(\geq 6: 69 \%)$ \\
\hline MCTA-A1-ASPECTS & $5.0 \pm 0.6(\geq 6: 47 \%)$ \\
\hline Collateral grading & $\begin{array}{l}0: 0,1: 8,2: 8,3: 4,4 \text { (intermediate):6, } 5 \\
\text { (good):6 }\end{array}$ \\
\hline $\begin{array}{l}\text { Predicted occlusion segment on } \\
\text { mCTA-A1 }\end{array}$ & C1:24, C2:4, C3:1, C4:2, C5:1, C6:0, C7:0 \\
\hline $\begin{array}{l}\text { Predicted occlusion segment on } \\
\text { mCTA-A3 }\end{array}$ & $\mathrm{C} 1: 18, \mathrm{C} 2: 4, \mathrm{C} 3: 3, \mathrm{C} 4: 3, \mathrm{C} 5: 2, \mathrm{C} 6: 2, \mathrm{C} 7: 0$ \\
\hline $\begin{array}{l}\text { Contrast migration } \geq 1 \text { segment } \\
\text { from mCTA-A1 to mCTA-A3 }\end{array}$ & $12(37.5)$ \\
\hline General anesthesia & $17(40.6)$ \\
\hline Occlusion segment on angiogram & C1:15, C2:2, C3:0, C4:4, C5:3, C6:4, C7:4 \\
\hline $\begin{array}{l}\text { Angioplasty for proximal cervical } \\
\text { ICA }\end{array}$ & $3(9.4)$ \\
\hline Suction & $2.3 \pm 0.3(0-6)$ \\
\hline Stent-retrieving & $0.8 \pm 0.2(0-4)$ \\
\hline Onset to reperfusion time, $\min$ & $338.5 \pm 15.7(215-594)$ \\
\hline mTICI, \% & 0:13, 1:1, 2a:2, 2b:7, 3:9 (2b-3: 50\%) \\
\hline
\end{tabular}

Abbreviations: ASPECTS, Alberta Stroke Program Early CT score; mCTA, multiphase computed tomographic angiography; mTICI, modified thrombolysis in cerebral infarction; SD, standard deviation; tPA, tissue plasminogen activator.

${ }^{\mathrm{a}}$ Values are expressed as mean $\pm \mathrm{SD}$ (range) or No. (\%).

Table 3. Angiographic Occlusion Segment at C6-7 Versus C1-5

\begin{tabular}{lccc}
\hline \multirow{2}{*}{ Variable } & \multicolumn{3}{c}{ Group (case number) } \\
\cline { 2 - 4 } & C6-7 $(\mathbf{n}=\mathbf{8})$ & C1-5 $(\mathbf{n}=\mathbf{2 4})$ & P value \\
\hline NIHSS & $19.3 \pm 4.7(12-26)$ & $18.4 \pm 5.4(8-30)$ & 0.701 \\
\hline ASPECTS & $7.9 \pm 2.3(4-10)$ & $8.8 \pm 1.4(5-10)$ & 0.296 \\
\hline mCTA-Pre-ASPECTS & $7.1 \pm 2.4(4-10)$ & $7.0 \pm 2.8(0-10)$ & 0.941 \\
\hline mCTA-A1-ASPECTS & $2.5 \pm 2.3(0-6)$ & $5.9 \pm 3.4(0-10)$ & $0.015^{\mathrm{b}}$ \\
\hline Collateral grading & $2.4 \pm 1.3(1-4)$ & $3.0 \pm 1.6(1-5)$ & 0.346 \\
\hline Good collateral & $0(0.0)$ & $6(25.0)$ & $0.011^{\mathrm{b}}$ \\
\hline mTICI (2b-3) & $6(75.0)$ & $10(41.7)$ & 0.109 \\
\hline 3-month-mRS $(\mathbf{0}-2)$ & $3(37.5)$ & $3(12.5)$ & 0.233 \\
\hline
\end{tabular}

Abbreviations: ASPECTS, Alberta Stroke Program Early CT score; mCTA, multiphase computed tomographic angiography; mRS, modified Rankin scale; mTICI, modified thrombolysis in cerebral infarction; NIHSS, National Institutes of Health Stroke scale; SD, standard deviation.

${ }^{a}$ Values are expressed as mean \pm SD (range) or No. (\%)

${ }^{\mathrm{b}}$ Pvalue $<0.05$

contrast stasis group $(6.1 \pm 3.5$, range: $0-10)$, with a p-value of 0.029 . Second, the contrast migration group had fewer patients with good collateral ( $0 \%)$, compared with the contrast stasis group (30\%), with a P value of 0.01 .

Furthermore, the results of the patients requiring an- gioplasty for concurrent proximal cervical ICA stenosis were compared to the rest of the patients. More patients had good ASPECTS (scores of 6 to 10) in the pre-contrast images of the multiphase CTA (100\% vs $65.5 \%$, P value $=0.001)$, but fewer patients had a good collateral (0\% vs $20.7 \%$, P value $=0.012$. Though there were more people in the angioplasty group with good mTICI results (2b and 3 ) $(100 \%$ vs $44.8 \%$, P value $<0.001)$, there were fewer patients that had a good 3-month mRS score (0 to 2) ( $0 \%$ vs $20.7 \%$, P value $=0.012$ ).

\section{Discussion}

Acute stroke cases with ICA occlusions display relatively fewer good outcomes (3-month mRS $0-2: 19 \%$ of patients) and reperfusion rates (mTICI 2b-3: 50\%) compared to previous studies, which are mainly composed of middle cerebral artery occlusion cases. There were 18 cases with contrast stasis at the $\mathrm{C} 1$ segment of the ICA on the multiphase CTA, but among them, only 15 cases had an angiographic occlusion at the $\mathrm{C} 1$ segment and three of them required a balloon angioplasty for a concurrent proximal cervical ICA tight stenosis. Compared to proximal ICA occlusions at $\mathrm{C} 1$ to $\mathrm{C} 5$, those with occlusions at the $\mathrm{C} 6$ to $\mathrm{C} 7$ segments demonstrated lower ASPECTS on A1 phase images and fewer patients had a good collateral on the multiphase CTA. However, there were no significant differences in their mTICI grades and 3-month mRS scores.

The original five large studies supporting the efficacy of IAT in acute stroke patients with a large vessel occlusion demonstrated good outcomes in $46.0 \%$ of patients, which is significantly higher than the $26.5 \%$ in the control arm. In addition, successful revascularization with an mTICI score of $2 \mathrm{~b}$ or 3 occurred in $71 \%$ (7). Compared to these results, there was a relatively lower rate of good outcomes (19\%) and successful reperfusion rates (50\%) for ICA occlusion cases in our study. According to the literature, there are several studies with different treatment combinations for acute strokes using ICA occlusion, and the results show that intravenous thrombolytic therapy alone achieves favorable outcomes in $0 \%-32.1 \%$ of patients. Meanwhile, when combined with intra-arterial therapies including thrombolysis, angioplasty, stents, stent-retrievers, and aspiration, $24 \%-100 \%$ have a favorable outcome, and $38 \%$ - $100 \%$ have a successful reperfusion rate (9-11). Though the results of previous studies are diverse, our results are not significantly different, but we did see slightly fewer favorable outcomes. However, the threshold of a successful reperfusion ranged from at least a TICI of 2 in order to successfully complete reperfusion, which might have caused some variation. Our definition of favorable outcome is relatively consistent with the literature, but some studies 
have different definitions, such as an mRS 0 - 2 at 1 month, or a Barthel index > 90, or a NIHSS score of $0-1$ at 3 months. Nonetheless, recent meta-analysis studies still suggest IAT is a favored treatment in acute strokes with ICA occlusions $(7,12)$.

In multiphase CTA, there were 18 cases that showed contrast stasis at the $\mathrm{C} 1$ segment. However, the angiography confirmed that only 15 of them had a true occlusion at the $\mathrm{C} 1$ segment, and three of them had a concurrent tight stenosis at the proximal cervical ICA. A concurrent tight stenosis can be identified by the presence of different conditions. Extracranial carotid disease accounts for $20 \%$ cases of acute ischemic stroke, and $10 \%$ of these cases present with acute occlusions. However, acute ischemic stroke with extracranial carotid disease is usually associated with ipsilateral intracranial occlusions, and there were only $8 \%$ cases of acute ischemic stroke purely due to carotid disease (13). Atherosclerosis is the main cause of internal carotid stenosis, and more commonly involves bifurcation (14). Though less frequently encountered, the occlusion site at the proximal ICA near the bifurcation location should be considered, because this location is the most common ICA location affected by atherosclerosis. The possibility of this condition is higher when the multiphase CTA suggests occlusion at the $\mathrm{C} 1$ segment. In our study, the condition accounts for only $9.4 \%$ of overall ICA occlusions, but increased to $16.7 \%$ in the predicted $\mathrm{C} 1$ segment occlusions. The treatment procedure is different for IAT when a tight stenosis at the proximal cervical ICA exists. Neither aspiration nor stent-retrieving will re-open the stenosis. For all three cases in this study, aspiration was first conducted, but the follow-up angiography revealed that the same occlusion was visible even after aspirating 2 or 3 times. Therefore, we use a balloon angioplasty to re-open the suspected concurrent tight stenosis, and all of them showed excellent patency for the whole ICA course after the angioplasty. The percentage of occlusion at the concurrent proximal cervical ICA tight stenosis increases to $16.7 \%$ when the multiphase CTA predicts the occlusion site at the $\mathrm{C} 1$ level. We think the correctly predicted percentage can be further enhanced if the A2 and A3 phases of the multiphase CTA extend the scan range down to the carotid bifurcation, because most of our cases did not reveal the contrast enhancement level in the $\mathrm{A} 2$ and $\mathrm{A} 3$ phases. If the thrombus occlusion at the $\mathrm{C} 1$ segment is not combined with a proximal cervical ICA tight stenosis, the thrombus location is usually at the middle or distal portions of the $\mathrm{C} 1$ segment, and the contrast in the multiphase CTA will migrate distally from the A1 to A3 images although maybe only a short distance. In the original invention of multiphase CTA, the scan range in both the A2 and A3 phase is from the skull base to the vertex (8). However, the carotid bifurcation is commonly located below the skull base. According to a previous study, the most commonly used references for carotid bifurcations are the $\mathrm{C} 3 / 4$ intervertebral disc, $\mathrm{C} 4$ vertebral body, and the laminae of the thyroid cartilage (15). There are $87.5 \%$ - $100 \%$ cases showing the carotid bifurcation above the inferior margin of the $C 4$ vertebral body (16). Currently, the speed of the CT scan is enough to cover the scan range from the $\mathrm{C} 4$ vertebral body to the vertex within an 8-second interval, in order to fulfill the requirement of the multiphase CTA (8). It will be beneficial to extend the multiphase CTA scan range, in order to early detect the less common causes of acute strokes due to acute occlusions of a proximal cervical ICA tight stenosis. Early identification of this condition will hasten the procedure of IAT by trying balloon angioplasty as early as possible. Pseudoocclusion of ICA on single-phase CTA has been studied before, and the authors concluded that single-phase CTA is not reliable to distinguish true occlusions from pseudoocclusions and that there are up to $15 \%-32.4 \%$ cases with pseudo-occlusion. The differentiation between true occlusion and pseudo-occlusion is crucial, because the planning of the endovascular intervention and treatment for acute strokes might change $(17,18)$. We think a multiphase CTA with extended scan range on the $\mathrm{A} 2$ and $\mathrm{A} 3$ phases might be a potential solution to rule out pseudo-occlusion based on CTA findings, but further well-designed studies for this issue are required.

Previous studies suggest that the prognosis is relatively poor in acute strokes with tandem lesions at the ICA and middle cerebral artery, as compared to isolated ICA lesions (10). Distal ICA occlusions at the T-zone also shows less favorable outcomes $(24.8 \%$ - $28.5 \%)$ compared to proximal ICA occlusions (43.5\% - 46.0\%). However, in our study, we compared the results of the occlusion sites at the C1-5 segments with those at the C6-7 segments, and there were no significant differences in the mTICI grades or 3-month mRS scores. There were, however, significantly lower ASPECTS on A1 phase images, and fewer patients with good collateral in the group with occlusions at C6-7. The same results were also noted when we compare the contrast migration group with the contrast stasis group in the multiphase CTA. The positive correlation between the contrast migration and C6-7 occlusion was statistically significant $\left(\mathrm{R}^{2}=0.492, \mathrm{P}=0.006\right)$ after controlling for age and sex. Therefore, whether there is contrast migration is helpful to predict the occlusion site. Anatomically, when the occlusion side is located at the C6-7 segment, there is predictable effect on the collateral supply: the collateral from the contralateral anterior circulation will be blocked by the occlusion of the A1 and ICA junction, and the collateral from the posterior circulation is also blocked by the occlusion of the ipsilateral posterior communicating artery; there- 
fore, it is reasonable to say that distal occlusion of the ICA will cause a poor collateral and lower ASPECTS. In our study, there were significantly lower ASPECTS in the A1 phases but not in the non-enhanced CTs or pre-contrast images of the multiphase CTAs. This result is supported by previous studies that suggest the prediction power of infarction scores based on ASPECTS are better on CTA images than on nonenhanced CT images (19). Our results do not show a significant difference in clinical outcomes between C6-7 occlusions and C1-5 occlusions, which might be due to the limitation of having a small number of cases.

For patients receiving angioplasty for a concurrent proximal cervical ICA stenosis, more of these patients had good ASPECTS on pre-contrast images of multiphase CTA, but fewer had a good collateral or good clinical outcome. The results are questionable because these cases are related to proximal ICA occlusions but do not present similar results, likely due to the extremely small number of cases. Successful reperfusion occurred in $100 \%$ of these patients after their angioplasty, reflecting the importance of the detecting coexisting proximal cervical ICA tight stenosis, and the proper usage of balloon angioplasty. The existence of proximal cervical ICA tight stenosis raises concern that there might be a high incidence of coexisting intracranial tandem lesions; Moreover, which lesion should be managed first is controversial. There is supportive evidence for both anterograde and retrograde treatments with carotid stenting, but a new modified Dotter technique shows results that are more promising using catheter dilatation for the carotid stenosis. As a result, the IAT procedure will not be prolonged by the stenting procedure and the routine antiplatelet therapy after stenting can be avoided. This is important because an acute infarction with a large vessel occlusion is at risk for subsequent intracranial hemorrhages $(9,20)$. Our procedure followed this concept, but we still used a balloon angioplasty to achieve a better lumen width, because proximal occlusion patency is also helpful for brain perfusion (21).

There are limitations in this study. The case number was small, which might affect the statistical significance and the interpretation of the results. In addition, the study design was retrospective and therefore, there are heterogeneities about patients' conditions, usage of devices, technical steps of IAT, and the experience of the neurointerventionalists. In the future, a prospective study with a larger case number, a standardized procedure, and more specific patient selection criteria would be helpful to confirm the results of this study.

In conclusion, acute stroke cases with ICA occlusions display a relatively lower percentage of good outcomes and reperfusion rates when compared to previous studies, which have mainly studied middle cerebral artery oc- clusions. Distal occlusion of the ICA demonstrates lower ASPECTS on the A1 phase images, and fewer patients had a good collateral on the multiphase CTA. Multiphase CTA with an extended scan range on the $\mathrm{A} 2$ and $\mathrm{A} 3$ phases can potentially rule out pseudo-occlusion of the ICA. Balloon angioplasty is key to achieving a high successful reperfusion rate when there is a concurrent proximal cervical ICA tight stenosis with an acute occlusion.

\section{Acknowledgments}

We would like to thank all the medical team members involved in stroke management at the Taipei Hospital, Tamsui Mackay Memorial Hospital, and Chiayi Chang Gung Memorial Hospital, particularly the Departments of Radiology, Neurology, and Neurosurgery in all these hospitals.

\section{Footnotes}

Authors' Contributions: Chun-Chao Huang wrote thisar- ticle and reviewed the imaging exams. Wei-Ming Huang and Zong-Yi Jhou performed statistical analysis. Yung-Pin Huang, Hsin-Yao Lin and Yuan-Hsiung Tsai collected clinical data. Chao-Liang Chou and Chun-Hsien Lin designed the study.

Conflict of Interests: The authors declare that they have no conflict of interest.

Ethical Approval: All procedures performed in the studies involving human participants were in accordance with the ethical standards of the institutional and/or national research committee and with the 1964 Helsinki Declaration and its later amendments or comparable ethical standards.

Funding/Support: This research did not receive any specific grants from funding agencies in the public, commercial, or not-for-profit sectors.

\section{References}

1. Berkhemer OA, Fransen PS, Beumer D, van den Berg LA, Lingsma $\mathrm{HF}$, Yoo AJ, et al. A randomized trial of intraarterial treatment for acute ischemic stroke. N Engl J Med. 2015;372(1):11-20. doi:10.1056/NEJMoa1411587. [PubMed: 25517348].

2. Goyal M, Demchuk AM, Menon BK, Eesa M, Rempel JL, Thornton J, et al. Randomized assessment of rapid endovascular treatment of ischemic stroke. N Engl J Med. 2015;372(11):1019-30. doi: 10.1056/NEJMoa1414905. [PubMed: 25671798].

3. Saver JL, Goyal M, Bonafe A, Diener HC, Levy EI, Pereira VM, et al. Stent-retriever thrombectomy after intravenous t-PA vs. t-PA alone in stroke. NEngl JMed.2015;372(24):2285-95. doi:10.1056/NEJMoa1415061. [PubMed: 25882376]. 
4. Campbell BC, Mitchell PJ, Kleinig TJ, Dewey HM, Churilov L, Yassi $\mathrm{N}$, et al. Endovascular therapy for ischemic stroke with perfusionimaging selection. N Engl J Med. 2015;372(11):1009-18. doi: 10.1056/NEJMoa1414792. [PubMed: 25671797].

5. Jovin TG, Chamorro A, Cobo E, de Miquel MA, Molina CA, Rovira A, et al. Thrombectomy within 8 hours after symptom onset in ischemic stroke. N Engl J Med. 2015;372(24):2296-306. doi: 10.1056/NEJMoa1503780. [PubMed: 25882510].

6. Powers WJ, Rabinstein AA, Ackerson T, Adeoye OM, Bambakidis NC, Becker K, et al. 2018 guidelines for the early management of patients with acute ischemic stroke: A guideline for healthcare professionals from the American Heart Association/American Stroke Association. Stroke. 2018;49(3):e46-e110. doi: 10.1161/STR.0000000000000158. [PubMed: 29367334].

7. Goyal M, Menon BK, van Zwam WH, Dippel DW, Mitchell PJ, Demchuk AM, et al. Endovascular thrombectomy after large-vessel ischaemic stroke: A meta-analysis of individual patient data from five randomised trials. Lancet. 2016;387(10029):1723-31. doi: 10.1016/S01406736(16)00163-X. [PubMed: 26898852].

8. Menon BK, d'Esterre CD, Qazi EM, Almekhlafi M, Hahn L, DemchukAM, et al. Multiphase CT angiography: A new tool for the imaging triage of patients with acute ischemic stroke. Radiology. 2015;275(2):510-20. doi: 10.1148/radiol.15142256. [PubMed: 25633505].

9. Li W, Yin Q, Xu G, Liu X. Treatment strategies for acute ischemic stroke caused by carotid artery occlusion. Interv Neurol. 2016;5(34):148-56. doi:10.1159/000445304. [PubMed: 27781043]. [PubMed Central: PMC5075799].

10. Gliem M, Lee JI, Barckhan A, Turowski B, Hartung HP, Jander S. Outcome and treatment effects in stroke associated with acute cervical ICA occlusion. PLoS One. 2017;12(1). e0170247. doi: 10.1371/journal.pone.0170247. [PubMed: 28081270]. [PubMed Central: PMC5231377].

11. Kappelhof M, Marquering HA, Berkhemer OA, Majoie CB. Intraarterial treatment of patients with acute ischemic stroke and internal carotid artery occlusion: A literature review. J Neurointerv Surg. 2015;7(1):8-15. doi: 10.1136/neurintsurg-2013-011004. [PubMed: 24385555].

12. Badhiwala JH, Nassiri F, Alhazzani W, Selim MH, Farrokhyar F, Spears J, et al. Endovascular thrombectomy for acute ischemic stroke: A meta- analysis. JAMA. 2015;314(17):1832-43. doi: 10.1001/jama.2015.13767. [PubMed: 26529161].

13. Tallarita T, Lanzino G, Rabinstein AA. Carotid intervention in acute stroke. Perspect Vasc Surg Endovasc Ther. 2010;22(1):49-57. doi: 10.1177/1531003510380469. [PubMed: 20798079].

14. Samuel KC. Atherosclerosis and occlusion of the internal carotid artery. J Pathol Bacteriol. 1956;71(2):391-401. doi: 10.1002/path.1700710214. [PubMed: 13398885].

15. Mirjalili SA, McFadden SL, Buckenham T, Stringer MD. Vertebral levels of key landmarks in the neck. Clin Anat. 2012;25(7):851-7. doi: 10.1002/ca.22124. [PubMed: 22836507].

16. [No author listed]. Anatomical variations of the common carotid artery bifurcations in relation to the cervical vertebrae in ethiopia. Anat Physiol. 2013;4(3). doi: 10.4172/2161-0940.1000143.

17. Diouf A, Fahed R, Gaha M, Chagnon M, Khoury N, Kotowski M, et al. Cervical internal carotid occlusion versus pseudo-occlusion at ct angiography in the context of acute stroke: An accuracy, interobserver, and intraobserver agreement study. Radiology. 2018;286(3):1008-15. doi: 10.1148/radiol.2017170681. [PubMed: 29072979].

18. Akpinar S, Gelener P, Yilmaz G. Aetiologies of internal carotid artery pseudo-occlusions in acute stroke patients: What neurointerventionalists can expect. Br J Radiol. 2017;90(1070):20160352. doi: 10.1259/bjr.20160352. [PubMed: 27995806]. [PubMed Central: PMC5685129].

19. Camargo EC, Furie KL, Singhal AB, Roccatagliata L, Cunnane ME, Halpern EF, et al. Acute brain infarct: Detection and delineation with CT angiographic source images versus nonenhanced CT scans. $R a$ diology. 2007;244(2):541-8. doi: 10.1148/radiol.2442061028. [PubMed: 17581888].

20. Woodward K, Wegryn S, Staruk C, Nyberg EM. The Dotter method revisited: Early experience with a novel method of rapid internal carotid artery revascularization in the setting of acute ischemic stroke. J Neurointerv Surg. 2016;8(4):360-6. doi: 10.1136/neurintsurg2014-011587. [PubMed: 25636305].

21. Gao F, Joyce Lo W, Sun X, Xu X, Miao Z. Combined use of stent angioplasty and mechanical thrombectomy for acute tandem internal carotid and middle cerebral artery occlusion. Neuroradiol J. 2015;28(3):316-21. doi: 10.1177/1971400915591679. [PubMed: 26246102]. [PubMed Central: PMC4757296]. 